\title{
Defective twin boundaries in nanotwinned metals
}

\author{
Y. Morris Wang ${ }^{1 \star}$, Frederic Sansoz ${ }^{2}$, Thomas LaGrange ${ }^{1}$, Ryan T. Ott' ${ }^{3}$, Jaime Marian', \\ Troy W. Barbee Jr ${ }^{1}$ and Alex V. Hamza
}

\begin{abstract}
Coherent twin boundaries (CTBs) are widely described, both theoretically and experimentally, as perfect interfaces that play a significant role in a variety of materials. Although the ability of CTBs in strengthening, maintaining the ductility and minimizing the electron scattering is well documented ${ }^{1-3}$, most of our understanding of the origin of these properties relies on perfect-interface assumptions. Here we report experiments and simulations demonstrating that as-grown CTBs in nanotwinned copper are inherently defective with kink-like steps and curvature, and that these imperfections consist of incoherent segments and partial dislocations. We further show that these defects play a crucial role in the deformation mechanisms and mechanical behaviour of nanotwinned copper. Our findings offer a view of the structure of CTBs that is largely different from that in the literature ${ }^{2,4,5}$, and underscore the significance of imperfections in nanotwinstrengthened materials.
\end{abstract}

CTBs formed during growth, deformation or annealing exist broadly in many crystalline solids with low or medium stackingfault energies ${ }^{1,5,6}$. The strengthening behaviour and other attractive properties of CTBs have been studied in nanotwinned metals (with an average twin spacing $<100 \mathrm{~nm}$; refs $7-9$ ). One prevalent view is that CTB-strengthened materials have certain advantages over nanocrystalline or ultrafine-grained materials; that is, materials strengthened through traditional grain boundaries (GBs) that are considered incoherent and defective ${ }^{10}$. GBs not only scatter electrons, but can migrate and slide under shear stresses ${ }^{11}$, leading to a maximum in strength in nanocrystalline materials ${ }^{12,13}$. In contrast, such migration/sliding mechanisms may not be operative in CTBs despite some reports of detwinning evidence $e^{7,14,15}$ and the observation of a similar maximum strength in a nanotwinned copper ${ }^{3}$ (nt-Cu). Existing models widely assume perfect CTBs and rationalize flow softening due to CTB migrations and detwinning as caused by nucleation and motion of partial dislocations parallel to CTBs (ref. 4). These mechanisms are informative as long as CTB lengths are limited to the tens of nanometres typically used in molecular dynamics simulations ${ }^{4,16-18}$. It still remains difficult through molecular dynamics simulations to validate the migrations/detwinning of the much longer CTBs seen in experiments (500 nm; ref. 3). There could be alternative mechanisms that are intricately related to the potential structures of CTBs and the characteristics of GBs, both of which are not accounted for in the literature.

Recent studies of nanotwinned copper pillars without GBs revealed strong deformation anisotropy and a brittle-to-ductile transition behaviour (where CTBs are considered intrinsically brittle $)^{2}$, suggesting that CTBs alone are not sufficient for increased plasticity despite their strong strengthening effect, and that a reasonable mix of GBs is helpful to mediate the plasticity and achieve high ductility. Experiments and simulations have frequently quoted GBs as one primary source of dislocations in nanotwinned polycrystalline materials, the nature of which is expected to have impacts on the mechanical behaviour; that is, experimental evidence hints at the relevance of GBs in nanotwinned metals. Unfortunately, limited information is available regarding the nature of GBs and the roles they are playing in controlling the plasticity.

We have examined the nanometre structural features of many twin boundaries (TBs) and the characteristics of GBs using a recently developed inverse pole figure orientation mapping (IPFOM) in a field-emission transmission electron microscope (TEM). This nanodiffraction-based technique has a spatial resolution of $\sim 1 \mathrm{~nm}$ that is comparable to the conical-scanning dark-field imaging approach $^{19}$. In a standard IPFOM image, 250,000 frames of diffraction patterns were collected, each of which was indexed by EDAX orientation-image-mapping indexing software and applied to determine the crystallographic orientations. The orientationresolving ability of IPFOM allows us to identify many previously unobserved microstructural defect features on preconceived-to-be completely perfect TBs. The materials used in our studies are nt-Cu synthesized by magnetron sputtering (see Methods and Supplementary Information). Shown in Fig. 1a is the cross-sectional microstructure of an as-deposited material, indicating a twin spacing of $\sim 5-70 \mathrm{~nm}$ with grain column width spanning from $\sim 0.3$ to $1.8 \mu \mathrm{m}$. Most TBs appear rather straight without obvious evidence of defective structures. Similar materials have been synthesized in parallel by other groups albeit with clear variations in twin density and columnar grain size ${ }^{14,20}$. To obtain statistical misorientations of TBs and GBs, we carried out detailed IPFOM studies in TEM using the NanoMegas Astar system ${ }^{21}$ (the pixellation effect of this technique can be seen in Supplementary Figs S1 and S2). As shown in Fig. 1b, under low resolution the as-synthesized nt-Cu contains at least two types of TB; that is, $\Sigma 3\{111\}$ CTBs normal or inclined to the growth direction that often transverse the columns, and a very limited number of $\Sigma 3\{112\}$ incoherent TBs (ITBs) that tend to bond CTBs terminated inside grains (marked with circles). Owing to their low frequency, the influence of these ITBs on the mechanical behaviour is considered inconsequential until the twins become very thin (<2 nm; ref. 17). However, a zoomed-in high-resolution IPFOM image shown in Fig. 1c indicates that originally thought perfect $\Sigma 3\{111\}$ CTBs contain many kink-like steps along the interfaces (a few are marked with arrows). These steps are incoherent $\Sigma 3\{11 \overline{2}\}$ segments, as evidenced by their crystallographic orientations, and their heights $(\sim 1-5 \mathrm{~nm})$ are visibly smaller than the ITBs mentioned above. The CTBs observed in IPFOM analysis are imperfect and comprise numerous partial dislocations, as compared with those with perfectly straight lines in two-dimensional images. For simplicity, we hereinafter refer to these steps as kinks in two-dimensional descriptions. Note that these nanometre features are substantially larger than the Burgers vector length $(0.147 \mathrm{~nm})$ of

\footnotetext{
${ }^{1}$ Physical and Life Sciences Directorate, Lawrence Livermore National Laboratory, Livermore, California 94550, USA, ${ }^{2}$ School of Engineering, The University of Vermont, Burlington, Vermont 05405, USA, ${ }^{3}$ Division of Materials Sciences and Engineering, Ames Laboratory (USDOE), Ames, lowa 50011, USA. *e-mail:ymwang@llnl.gov
} 

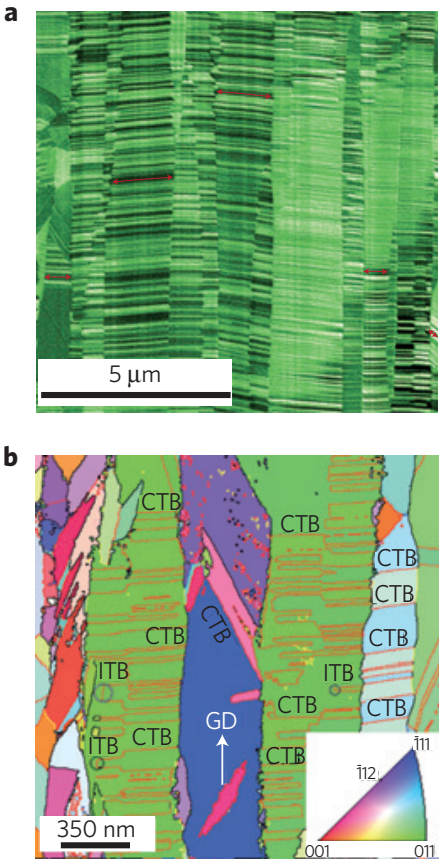

c

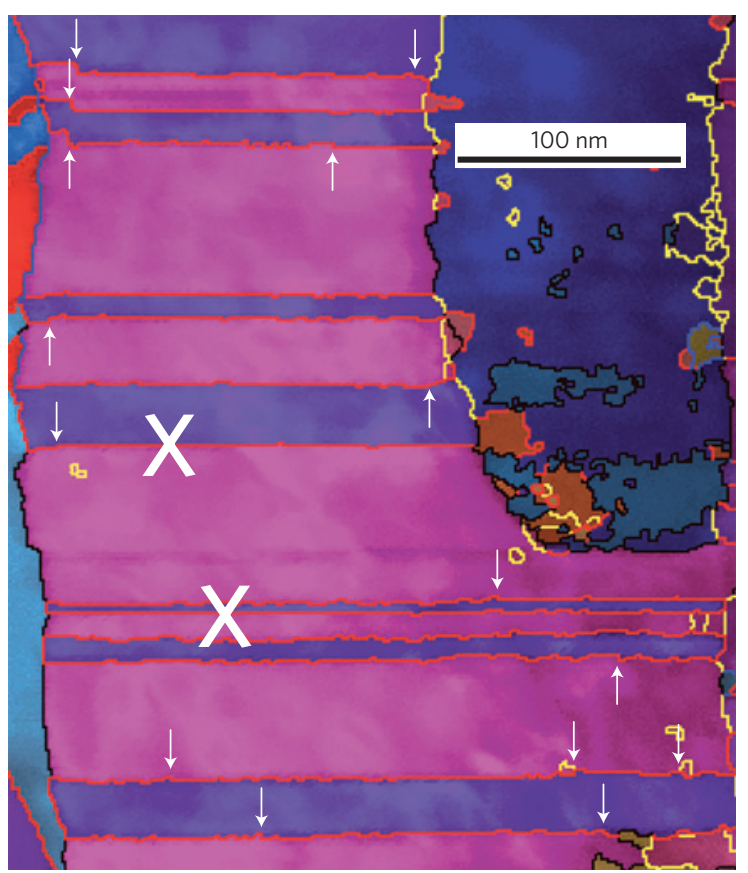

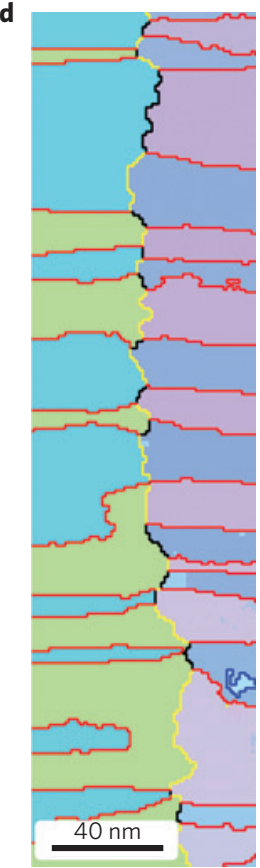

Figure 1 | Microstructure of as-grown nanotwinned copper. a, A focused-ion-beam ion-channelling image of the as-grown nanotwinned copper. The imaging condition is $30 \mathrm{keV}, 9.7 \mathrm{pA}$. The orientations of representative TBs are marked with double-headed red arrows. b, An IPFOM image of growth twins. The coherent and incoherent TBs are labelled as CTB and ITB (inside circles), respectively. The growth direction (GD) of the film is marked with an arrow. c, An edge-on high-resolution IPFOM image of CTBs. Some selected kinks are marked with white arrows. The portion of CTBs marked with an $X$ is perfect TBs without defects. Note that the TEM sample for IPFOM is prepared by electropolishing. d, An IPFOM image of a long GB (from top to the bottom), which shows mixed segments of low- and high-angle GBs. The GBs are characterized by a coincidence site lattice (CSL) model. The interface colour scheme in b-d is as follows: yellow, low-angle GBs with misorientation < 15; black, large-angle GBs; red, $\Sigma 3$ CSL boundary; blue, $\Sigma 9$ CSL boundary. The orientations of grains are coloured according to the inverse (001) pole figure shown in the inset of $\mathbf{b}$.

a single Shockley partial previously reported on CTBs (ref. 3); but the existence of these defective structures is not at odds with these earlier high-resolution TEM studies. A close examination of Fig. 1c suggests that the kink distributions are relatively non-uniform from TB to TB but that their density is rather persistent; that is, kink density per unit area increases with twin density. For the sample shown in Fig. 1c, there exists an average of one kink per 10-nm-long CTB (Supplementary Fig. S3). These findings strongly suggest that as with conventional GBs, many CTBs have defective structures (that is, kinks or incoherent components) that have not been considered in previous studies. The formation mechanisms of these kinks are not well understood; however, they seem to be the intrinsic characteristics of growth twins. It is conceivable that these features are related to processing or deposition conditions, and are expected to impact mechanical properties.

Another IPFOM image shown in Fig. 1d reveals that GBs in nt-Cu are more defective and full of partial-dislocation-like defects, as manifested by abundant kinks along the GBs. Interestingly, the statistical misorientation angle distribution maps obtained from both cross-section (Supplementary Fig. S4) and plan-view indicate an appreciable fraction of low-angle GBs (misorientation $<15^{\circ}$ ), leading to a GB network distribution that is far away from a MacKenzie distribution ${ }^{22}$ - the characteristic GB distribution of a well-annealed coarse-grained copper. Note that the curvy and defective nature of CTBs remains visible in this image. The GB distributions observed here strongly suggest that the classical Voronoi construction approach for GB networks in past modelling studies may not be applicable to nanotwinned materials reported here.

To help reveal the strain-dependent deformation mechanisms and the deformation anisotropy (copper has an anisotropic factor (A) of $3.21 ; A=2 C_{44} /\left(C_{11}-C_{12}\right)$, where $C_{11}, C_{12}$ and $C_{44}$ are elastic constants of copper)), we performed in situ synchrotron X-ray diffraction (SXRD) experiments at two different temperatures to track in real-time the elastic and plastic deformation of nt-Cu. In addition to offering a better statistical approach for bulk materials ${ }^{23}$, the in situ SXRD has the advantageous capability of resolving anisotropic microstrains ${ }^{24}$. Six crystallographic indices (that is, $111,200,220,311,222,400)$ were followed simultaneously in these in situ SXRD experiments. To investigate the microplasticity, we quantitatively calculate the lattice strain deviation as $\Delta \varepsilon^{h k l}=\varepsilon^{h k l}-\sigma / E^{h k l}$, where $\varepsilon^{h k l}=\left(d^{h k l}-d_{\mathrm{o}}^{h k l}\right) / d_{\mathrm{o}}^{h k l}\left(d_{\mathrm{o}}^{h k l}\right.$ and $d^{h k l}$ are the inter-planar spacing before and during loading, respectively), $\sigma$ is the applied stress and $E^{h k l}$ is the measured average elastic modulus for a specific plane $h k l$ (see Supplementary Table $\mathrm{S} 1$ ). Figure 2a,b shows the $\Delta \varepsilon^{h k l}$ behaviour parallel to the tensile axis (that is, longitudinal direction) for four representative Miller indices tested at room temperature and $180^{\circ} \mathrm{C}$ (see Supplementary Fig. S6 for the heating profile), respectively; the $\Delta \varepsilon^{h k l}$ behaviour for the transverse direction can be seen in Supplementary Fig. S7. For clarity, the corresponding tensile stress-strain curves are included, which show softening behaviour at both temperatures. In coarse-grained face-centred-cubic copper, the deformation is governed by full dislocation slips of $1 / 2\langle 110\rangle\{111\}$ type, leading to preferential yielding and negative $\Delta \varepsilon$ of 220 reflection at the onset of macroscopic plasticity, whereas 200 exhibits the largest positive $\Delta \varepsilon$ (ref. 25). Interestingly, the $\Delta \varepsilon$ trend in nt-Cu shows a rather different behaviour, where 200 shows a pronounced negative $\Delta \varepsilon$ before $0.2 \%$ yield stress, and sharply curls back to the positive territory on further loading. The 111 reflection exhibits similar curling behaviour; but to a smaller degree. By comparison, the $\Delta \varepsilon$ of 220 remains positive during the deformation. The initial negative deviation of $\Delta \varepsilon$ for both 200 and 111 reflections in $\mathrm{nt}-\mathrm{Cu}$ is an indication of preferable slip activations next to $\langle 001\rangle$ and $\langle 111\rangle$ orientations, suggestive of $1 / 6\langle 112\rangle\{111\}$ partial related 


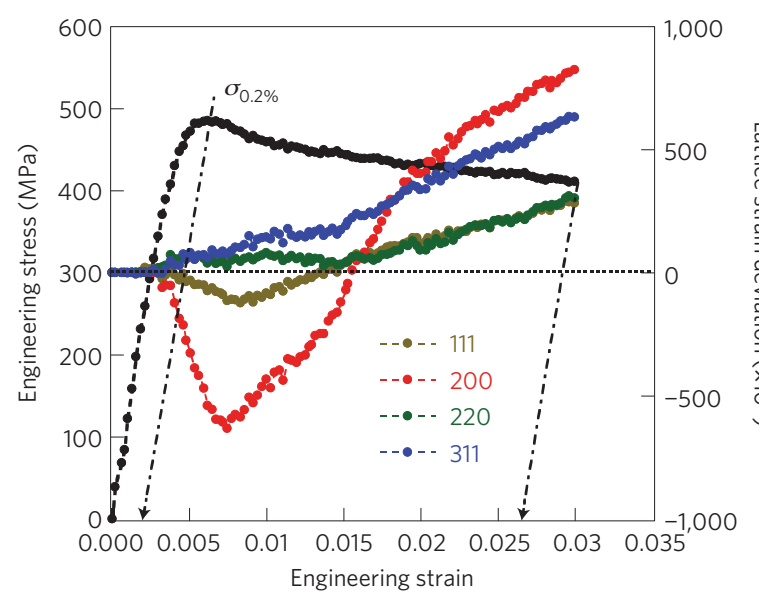

b

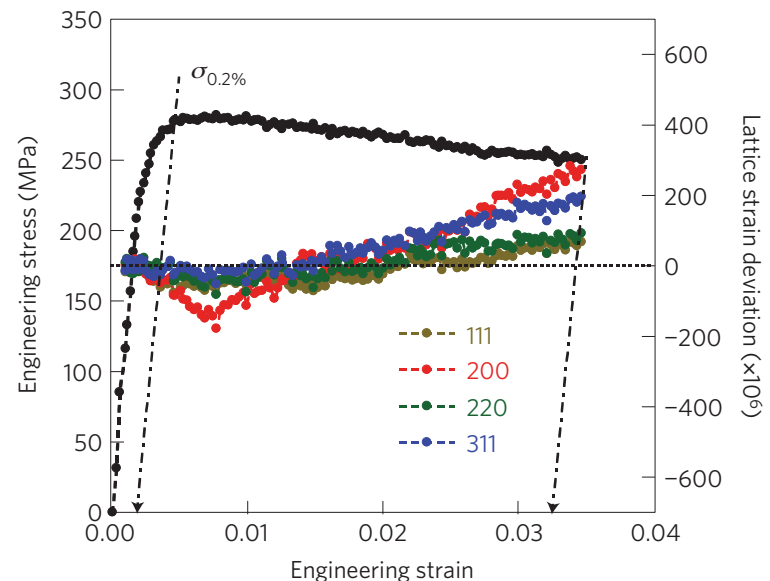

Figure $\mathbf{2}$ | Lattice strain deviation behaviour in tension. a,b, Engineering stress-engineering strain curves (black) and lattice strain deviation behaviour (coloured) of $\mathrm{nt}-\mathrm{Cu}$ along the longitudinal direction at room temperature (a) and $180^{\circ} \mathrm{C}(\mathbf{b})$. The $0.2 \%$ macroscopic yield stress is marked as $\sigma_{0.2 \%}$. The strain rate is $6 \times 10^{-5} \mathrm{~s}^{-1}$ for both temperatures. The plots show zoomed-in regions of the stress-strain curves corresponding to plastic strains of 0.027 and 0.033 for the samples deformed at room temperature and $180^{\circ} \mathrm{C}$, respectively.

deformation mechanisms (see the inverse pole figure shown in Fig. 1b, inset). The $\Delta \varepsilon$ behaviour further indicates that the microplasticity initiates well before the macroscopic yielding occurs in these materials, probably owing to some easy dislocation sources (such as those observed from IPFOM). Moreover, we find that the deformation of nt-Cu is sensitive to the temperature (Fig. 2b). The yield stress drops by $43 \%$ at $180^{\circ} \mathrm{C}$, the plastic deformation of which is clearly more isotropic as the difference of $\Delta \varepsilon$ among various crystallographic orientations becomes substantially smaller compared with room temperature.

The room-temperature peak broadening at different strain levels plotted according to the Williamson-Hall approach (Fig. 3) suggests that there is a significant amount of dislocation accumulation and the development of strong anisotropy during loading and after fracture, as evidenced by the increased Williamson-Hall slope and the continuously broadening of all peaks (for example, 311) vis-à-vis the as-deposited state ${ }^{26}$. A substantial portion of peak broadening remains permanent after fracture, in contrast to the reversible peak broadening and strong plastic recovery in nanocrystalline materials ${ }^{23,27}$. This behaviour generally agrees with the evolution of the inhomogeneous (root-mean-square) strain of nt- $\mathrm{Cu}$ and the texture development (Supplementary Fig. S8). The increased dislocation density during deformation and after

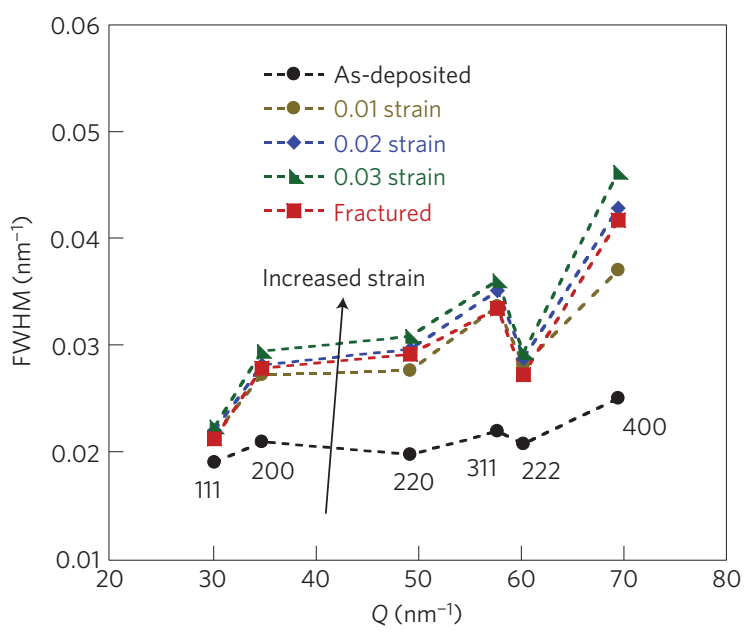

Figure 3 | Full-width at half-maximum (FWHM) as a function of the scattering vector $(Q)$ at various loading strains and after fracture (that is, Williamson-Hall plots). The scattering vector $Q=4 \pi \sin \theta / \lambda$, where $2 \theta$ is the angle between incident and diffracted beams, and $\lambda$ is the wavelength $(\mathrm{nm})$. These data are extracted from the profile fitting of 300 synchrotron spectra recorded during the entire tensile test at room temperature. The strain labelled in the figure includes macroscopic elastic and plastic components (except for the fractured sample).

fracture contrasts with the lack of strain hardening in this material, which may suggest other softening mechanisms that counterbalance the dislocation hardening. Post-mortem TEM examinations of the room-temperature-deformed sample shown in Fig. 4a indicate strong accumulations of dislocations between the TBs and inside the grains. TEM also reveals the disappearance of some growth twins (that is, detwinning) in certain regions at room temperature. At $180^{\circ} \mathrm{C}$, most $\mathrm{TBs}$ are annihilated, concomitant with grain coarsening (Fig. 4b). These TEM observations, together with in situ SXRD tensile results, offer some clues that these defective TBs are unstable against thermo-mechanical deformations.

Although the experimental observations reported above are qualitatively self-consistent, we speculate that the real atomic processes involved with these kinked TBs during deformation are quite complex. Therefore, molecular dynamics simulations using models built from kinked TBs and perfect TBs (Supplementary Fig. S9) are performed to explore this question. Two types of GB, smooth or defective, are also incorporated to tackle the effects of GB characteristics on mechanical behaviour; that is, GBs with either high or low yield stresses, respectively (Supplementary Fig. S10). Furthermore, owing to the highly idealized models (for example, the tensile axis is perfectly parallel to TBs when in tension and thus no critical resolved shear stress), we simulate the deformation behaviour of nt-Cu under both uniaxial tension and pure shear conditions, as the most likely loading scenario in the experiments is the mix of both due to the incline of TBs (Fig. 1a). From the simulations, we find that the nt-Cu models with kinked TBs have rather rich deformation processes that do not exist in the case of perfect TBs.

The stress-strain curves from different simulated microstructures deformed in tension are shown in Fig. 5a. The highest yield point and ultimate strength are observed in the sample with smooth GBs and perfect TBs, suggestive of difficult plastic deformation processes in these near-perfect samples. Threading dislocations are observed to emit from GBs and glide along slip directions parallel to TBs (Supplementary Table S2 and Supplementary Movie S1). Although this type of crystal slip has been considered as a hard mode in nt-Cu, with potential slip resistance owing to the constraints of small twin spacing on dislocation glide ${ }^{28}$, newly emitted threading dislocations are found to transmit easily without blockage through 

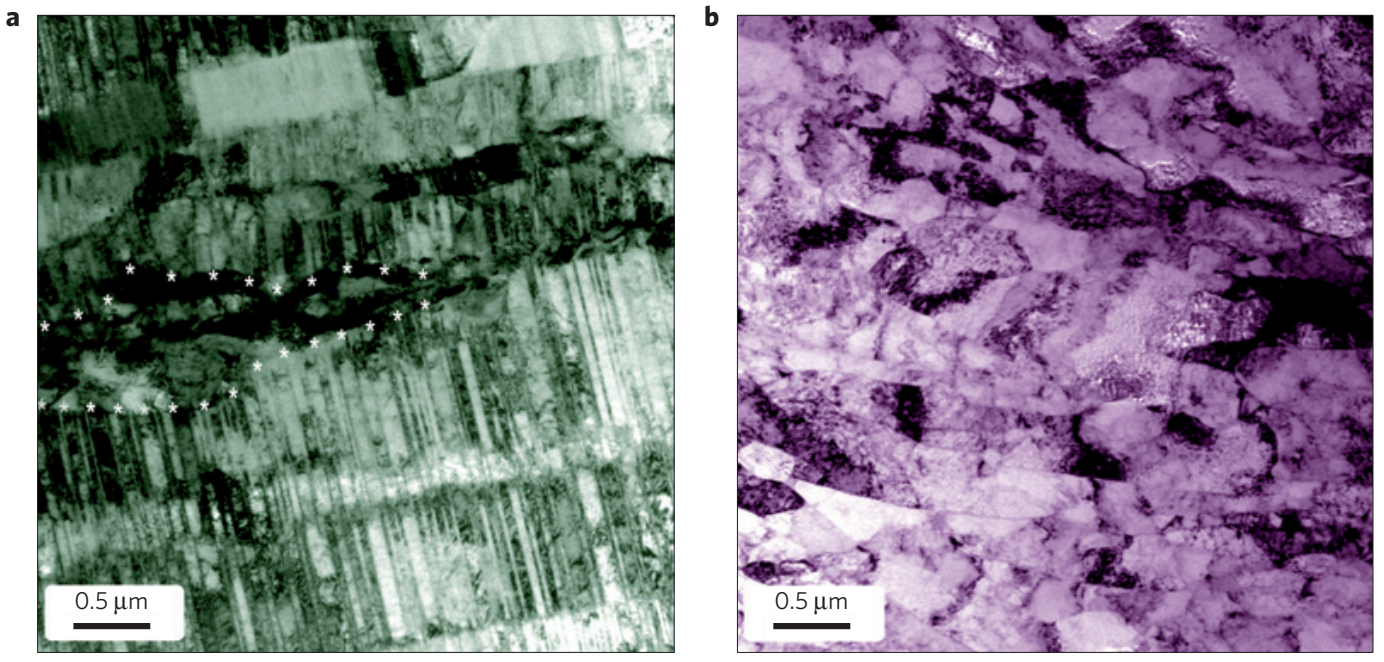

Figure 4 | Microstructure of post-mortem tensile samples. a,b, Bright-field TEM images of nt-Cu deformed at room temperature (a) and $180{ }^{\circ} \mathrm{C}(\mathbf{b})$. Note the loss of some growth twin structure in $\mathbf{a}$ (the region marked with asterisks), and the strong coarsening and complete disappearance of growth twins in $\mathbf{b}$
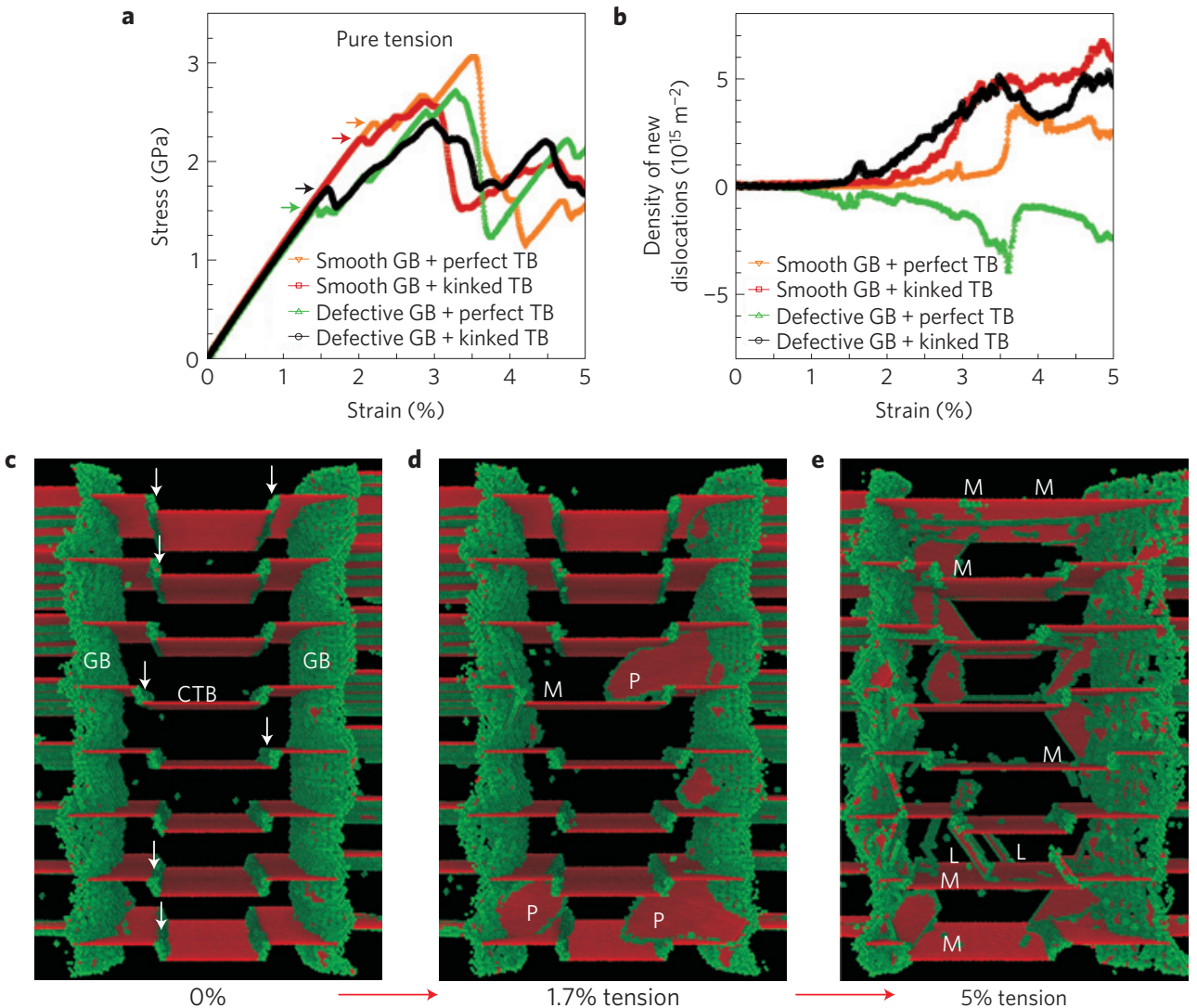

Figure 5 | Molecular dynamics simulations of deformation mechanisms in nt-Cu subjected to uniaxial tension with a twin spacing of $5 \mathrm{~nm}$.

a, Stress-strain curves for four idealized models containing either smooth or defective GBs, and perfect TBs or kinked TBs. Each arrow shows the onset of yielding. b. Density of new dislocations as a function of applied strain. c-e, Microstructure and deformation mechanisms in a columnar grain with both defective GBs and kinked TBs at different stages of deformation. Vertical arrows indicate the positions of migrating kink imperfections. P, partial dislocations emitted from GBs; M, kink migration; L, Lomer locks.

perfect TBs (ref. 29), and disappear in the opposite GB in the course of deformation (Supplementary Fig. S11 and Supplementary Movie S1). These mechanisms result in no increase, or even a decrease, in dislocation density in all models with perfect TBs (Fig. 5b). The inclusion of defective GBs (similar to those observed from experiments) substantially decreases the initial yield stress with no change in deformation mechanisms. The same effects of GB structure dominate the yield point of nt-Cu containing kink defects (Fig. 5a,c,d, Supplementary Table S2 and Supplementary Movie S2a). Remarkably, the introduction of kinks results in significant dislocation pinning giving rise to the formation of Lomer-Cottrell locks at the intersection of threading dislocations and kinks (Fig. 5c-e, Supplementary Discussion, Supplementary Fig. S12 and Supplementary Movie S2b). This kink-dependent mechanism is 
consistent with the net increase in dislocation density observed after initial yielding in our molecular dynamics simulations (Fig. 5b) and in situ SXRD experiments (Fig. 3). The simulations further indicate that these Lomer-Cottrell locks are short-lived and can be destroyed on further deformation. Furthermore, marked softening is observed at the stress peak (Fig. 5a and Supplementary Table S2) due to partial dislocations nucleated at kink-GB junctions, gliding along kink lines (Supplementary Fig. S13 and Supplementary Discussion). Concurrently, the pinning of threading dislocations decreases, as evidenced by the maximum in dislocation density reached in $\mathrm{nt}-\mathrm{Cu}$ with kinked TBs (Fig. 5b) because of kink migration, which also contributes to the softening behaviour. Motion of kinks closest to GBs is observable at the yield point, but becomes more important to the plastic flow at larger strains (Fig. 5e) because of the low resolved shear stress on kink steps in pure tension. We propose that the competition between Lomer-Cottrell lock formation at the initial yield point and kink-dependent softening mechanisms at higher stresses may have contributed to the change in 200 lattice strain deviation observed in in situ SXRD experiments (Supplementary Discussion).

Under pure shear stresses, the deformation of $\mathrm{nt}-\mathrm{Cu}$ becomes exclusively dominated by kink motion and migration of TBs (Supplementary Table S2, Supplementary Fig. S14 and Supplementary Movie S3). Interestingly, dislocations no longer nucleate from GBs but rather from kink ledges (Supplementary Fig. S14d and Supplementary Movie S3). The kinks are absorbed by GBs after migration, which leads to detwinning. The shear stress for kink migration is significantly smaller than that required to emit twinning partial dislocations from GBs in models with perfect TBs (Supplementary Fig. S14a,b and Supplementary Movie S4). These results demonstrate that shear stresses, such as those in the case of high-pressure torsion from the experiments ${ }^{30}$, favour kink migration and detwinning-a notion that bears surprising similarity to GB migrations ${ }^{11}$. The easy motion and migration of kinks under pure shear qualitatively agree with the early microscopic yielding of these materials and some detwinning seen in in situ SXRD and TEMs.

Despite the remaining open questions and nearly idealized approach of modelling, the combination of experimental and molecular dynamics results here requires that CTBs be treated as defective entities, in contrast to the traditional perfect-TB assumption. The migration of kinks is an emphatic sign that CTBs have intrinsic instability, notwithstanding experimental observations that they tend to be more stable than conventional GBs (refs 20,30). The migration of CTBs caused by kink motions suggests that CTBs close to GBs or longer CTBs with smaller spacing are more vulnerable to detwinning. This scenario is different from the detwinning process through partial dislocation glide proposed by modelling or from the detwinning caused by ITB motions observed in some experiments ${ }^{17,31}$. Nevertheless, the kink-motion proposition has strong implications to the detwinning phenomena observed so far experimentally (for example, detwinning remains active even at low temperatures and often occurs in certain regions $)^{7,14,15}$, as well as a maximum strength seen in nt-Cu (ref. 3). The detwinning near GBs is likely to help unlock triple junctions between GBs and TBs, facilitating GB migration and contributing further to the strain softening. For short CTBs of a few nanometres, a regime that is dominated by molecular dynamics simulations, the likelihood of generating kinks decreases, and CTBs can be considered quasi-perfect. On the other hand, the CTBs in the materials experimentally studied and reported on here are in the submicrometre range and observed to have inherent imperfections. The presence of such defective CTBs must be considered in both scientific research and practical utilities. We, in addition, believe that the attention being given to potential defects in CTBs resulting from annealing, deformation and various synthesis conditions is desirable to fully understand CTB-strengthened materials.

\section{Methods}

The nanotwinned copper samples were synthesized by a layer-by-layer magnetron sputtering technique. The details of sample conditions, twin density and geometry are given in the Supplementary Information. The intrinsic nanoscale structures of the as-synthesized CTB were characterized using an IPFOM technique in a TEM. The details of the IPFOM technique, resolution, potential pixellation effect, kink defect density, and additional high- and low-resolution IPFOM images are available in the Supplementary Information. The tensile properties of nanotwinned copper were characterized using an in situ SXRD approach performed at the Advanced Photon Source of Argonne National Laboratory (USA) at two different temperatures. Multiple nanotwinned samples were measured, and the in situ deformation information was extracted from the lattice strain deviations, peak profile and texture analysis (see more in the Supplementary Information). In addition, the post-mortem deformation microstructure was examined by conventional TEM. Multi-million atom molecular dynamics simulations were carried out using models built from experimental observations, where both perfect and defective twin boundaries were used, and the simulation results are compared with each other and to experimental findings. Further simulation details, results and movies are available in the Supplementary Information, together with a Supplementary Discussion on lattice strain deviation behaviour, additional deformation mechanisms, and the relevance of defective structures to the mechanical behaviour of nanotwinned copper.

Received 7 November 2012; accepted 2 April 2013; published online 19 May 2013

\section{References}

1. Zhu, Y. T., Liao, X. Z. \& Wu, X. L. Deformation twinning in nanocrystalline materials. Prog. Mater. Sci. 57, 1-62 (2012).

2. Jang, D. C., Li, X. Y., Gao, H. J. \& Greer, J. R. Deformation mechanisms in nanotwinned metal nanopillars. Nature Nanotech. 7, 594-601 (2012).

3. Lu, L., Chen, X., Huang, X. \& Lu, K. Revealing the maximum strength in nanotwinned copper. Science 323, 607-610 (2009).

4. Li, X. Y., Wei, Y. J., Lu, L., Lu, K. \& Gao, H. J. Dislocation nucleation governed softening and maximum strength in nano-twinned metals. Nature 464, 877-880 (2010).

5. Lu, K., Lu, L. \& Suresh, S. Strengthening materials by engineering coherent internal boundaries at the nanoscale. Science 324, 349-3652 (2009).

6. Yu, Q. et al. The nanostructured origin of deformation twinning. Nano Lett. 12, 887-892 (2012).

7. Shute, C. J. et al. Detwinning, damage and crack initiation during cyclic loading of $\mathrm{Cu}$ samples containing aligned nanotwins. Acta Mater. 659, 4569-4577 (2011).

8. Chen, K. C., Wu, W. W., Liao, C. N., Chen, L. J. \& Tu, K. N. Observation of atomic diffusion at twin-modified grain boundaries in copper. Science 321, 1066-1069 (2008).

9. Lu, L., Shen, Y. F., Chen, X. H., Qian, L. H. \& Lu, K. Ultrahigh strength and high electrical conductivity in copper. Science 304, 422-426 (2004).

10. Ovid'ko, I. A. Materials science-deformation of nanostructures. Science 295, 2386-2386 (2002).

11. Rupert, T. J., Gianola, D. S., Gan, Y. \& Hemker, K. J. Experimental observations of stress-driven grain boundary migration. Science 326, 1686-1690 (2009).

12. Schiotz, J. \& Jacobsen, K. W. A maximum in the strength of nanocrystalline copper. Science 301, 1357-1359 (2003).

13. Szlufarska, I., Nakano, A. \& Vashishta, P. A crossover in the mechanical response of nanocrystalline ceramics. Science 309, 911-914 (2005).

14. You, Z. S., Lu, L. \& Lu, K. Tensile behavior of columnar grained Cu with preferentially oriented nanoscale twins. Acta Mater. 59, 6927-6937 (2011).

15. Wang, Y. D. et al. Low temperature deformation detwinning-a reverse mode of twinning. Adv. Eng. Mater. 12, 906-911 (2010)

16. Wu, Z. X., Zhang, Y. W. \& Srolovitz, D. J. Deformation mechanisms, length scales and optimizing the mechanical properties of nanotwinned metals. Acta Mater. 59, 6890-6900 (2011).

17. Wang, J. et al. Detwinning mechanisms for growth twins in face-centered cubic metals. Acta Mater. 58, 2262-2270 (2010).

18. Stukowski, A., Albe, K. \& Farkas, D. Nanotwinned fcc metals: Strengthening versus softening mechanisms. Phys. Rev. B 82, 224103 (2010).

19. Liu, H. H. et al. Three-dimensional orientation mapping in the transmission electron microscope. Science 332, 833-834 (2011).

20. Bezares, J. et al. Indentation of nanotwinned fcc metals: Implications for nanotwin stability. Acta Mater. 60, 4623-4635 (2012).

21. Rauch, E. F., Rouvimov, S., Nicolopoulos, S. \& Moeck, P. High throughput automated crystal orientation and phase mapping of nanoparticles from HREM-TEM images. Microsc. Microanal. 15, 756-757 (2009).

22. Mackenzie, J. K. 2nd paper on statistics associated with the random disorientation of cubes. Biometrika 45, 229-240 (1958).

23. Budrovic, Z., Van Swygenhoven, H., Derlet, P. M., Van Petegem, S. \& Schmitt, B. Plastic deformation with reversible peak broadening in nanocrystalline nickel. Science 304, 273-276 (2004). 
24. Wang, Y. D. et al. The development of grain-orientation-dependentresidual stresses in a cyclically deformed alloy. Nature Mater. 2, 101-106 (2003).

25. Clausen, B., Lorentzen, T. \& Leffers, T. Self-consistent modelling of the plastic deformation of FCC polycrystals and its implications for diffraction measurements of internal stresses. Acta Mater. 46, 3087-3098 (1998).

26. Brandstetter, S., Derlet, P. M., Van Petegem, S. \& Van Swygenhoven, H. Williamson-Hall anisotropy in nanocrystalline metals: X-ray diffraction experiments and atomistic simulations. Acta Mater. 56, 165-176 (2008).

27. Rajagopalan, J., Han, J. H. \& Saif, M. T. A. Plastic deformation recovery in freestanding nanocrystalline aluminum and gold thin films. Science 315, 1831-1834 (2007).

28. You, Z. S. et al. Plastic anisotropy and associated deformation mechanisms in nanotwinned metals. Acta Mater. 61, 217-227 (2013).

29. Deng, C. \& Sansoz, F. Fundamental differences in the plasticity of periodically twinned nanowires in $\mathrm{Au}, \mathrm{Ag}, \mathrm{Al}, \mathrm{Cu}, \mathrm{Pb}$ and $\mathrm{Ni}$. Acta Mater. 57, 6090-6101 (2009).

30. Hodge, A. M. et al. Twin stability in highly nanotwinned $\mathrm{Cu}$ under compression, torsion and tension. Scr. Mater. 66, 872-877 (2012).

31. Xu, L. et al. Structure and migration of (112) step on (111) twin boundaries in nanocrystalline copper. J. Appl. Phys. 104, 113717 (2008).

\section{Acknowledgements}

The authors thank V. Bulatov and A. Stukowski for helpful discussions, and M. Besser, J. Almer, N. Teslich and R. Gross for experimental assistance. This work was performed under the auspices of the US Department of Energy (DOE) by Lawrence Livermore
National Laboratory under Contract No. DE-AC52-07NA27344, and Ames Laboratory (Office of Basic Energy Sciences) under Contract No. DE-AC02-07CH11358. The use of APS was supported by the US DOE under Contract No. DE-AC02-06CH11357. F.S. is grateful for support from the NSF CAREER program (grant DMR-0747658) and the computational resources provided by the Vermont Advanced Computing Centre (NASA grant NNX06AC88G). T.L. and IPFOM measurements are supported by US DOE, Office of Basic Energy Sciences. J.M. acknowledges financial support from the US DOE Early Career Research Program.

\section{Author contributions}

Y.M.W. and F.S. designed the experiments and simulations. T.W.B. synthesized the nanotwinned samples. Y.M.W., T.L. and R.T.O. performed experiments (TEM and FIB, IPFOM measurements, and in situ SXRD, respectively) and analysed the data. F.S. performed the simulations and analysis of deformation mechanisms. J.M contributed to the qualitative analysis of lattice strain deviation behaviour. Y.M.W. and F.S. wrote the manuscript with contributions from the other authors. All authors commented on the final manuscript and conclusions of this work.

\section{Additional information}

Supplementary information is available in the online version of the paper. Reprints and permissions information is available online at www.nature.com/reprints. Correspondence and requests for materials should be addressed to Y.M.W.

\section{Competing financial interests}

The authors declare no competing financial interests. 